\title{
11. Bemerkungen zu O. Lehmann: Die Kontinuität der Aggregatzustande und die flüssigen Kristalle ${ }^{1}$; von Karl Fuchs.
}

Hofrat Prof. Dr. O. Lehmann spricht im zitierten Aufsatz für die Annahme, daB die Molekule eines Stoffes in verschiedenen Aggregatzuständen, namentlich aber in verschiedenen Kristallsystemen (wenn der Stoff eben polymorph ist) verschiedenen Bau hätten. Gleichzeitig verwirft aber 0 . Lehmann auch die Raumgittertheorie, indem er darauf hinweist, daß in einem fließenden Kristall das Raumgitter fortwährend zerstört wird, ohne daB der Körper deswegen seine Kristalleigenschaften verlöre. Ich will auf Grund einer älteren Arbeit ${ }^{2}$ ) für die von O. Lehmann behauptete Polymorphie der Moleküle, aber auch für die von 0 . Lehmann verworfene Raumgittertheorie sprechen.

In meiner zitierten Arbeit ist der Gedanke ausgesprochen, daß ein Kristall nicht nur aus Einzelmolekülen, sondern auch aus Molekülkomplexen aufgebaut sein kann, und ein Stoff, der unter verschiedenen Umständen - etwa bei verschiedener Temperatur - verschiedene Molekülkomplexe von 1, 2, $3 \ldots$. Molekülen bildet, kann auch entsprechend in verschiedenen Systemen kristallisieren.

Die zitierte Arbeit geht von dem Grundsatze aus, daB in einem Kristall die Moleküle sich so lagern, daB die Molekularkräfte ein Maximum der Arbeit leisten; das Maximum können sie offenbar nur bei regelmäßiger Lagerung, also bei Lagerung nach irgend einem Raumgitter leisten. Daraus folgt aber, daB das Raumgitter sich immer unter positiver Arbeitsleistung rekonstruieren wird, wie oft man es auch stören oder zerstören mag, wenn die Moleküle nur, wie in einem flüssigen Kristalle,

1) O. Lehmann, Ann. d. Phys. 20. p. 77. 1906.

2) K. Fuchs, Beitrag zur Theorie der Kristallisation. Exners Rep. 2. p. 199. 1888 . 
genügend beweglich sind. Gleichwie eine Kiste, die man wälzt, immer wieder auf eine Fläche, also in eine Lage gröBter Arbeit schnappt, so schnappen auch im flieBenden Kristall die Moleküle immer wieder in die Gitterordnung gröBter Arbeit.

DaB eine solche fortwährende spontane Rekonstruktion eines gestörten Gitters praktisch möglich ist, das veranschaulicht ein einfacher Versuch. Wenn man in eine flache runde Schüssel eine Lage Schrotkörner tut, dann wollen alle Körner wegen der sphärischen Krümmung des Bodens nach der Mitte rollen. Die Schicht erstrebt also eine möglichst kleine Flächenausdehnung, als zögen die Kugeln, Molekülen gleich, einander an. Wenn man zur Erhöhung der Beweglichkeit die Schüssel dauernd in Vibration erhält, dann erscheint die Schicht sehr bald zusammengesetzt aus Feldern von dreiechig geordneten Kugeln, d. h. in irgend einem Felde ist jede Kugel von einem Kranz von sechs Kugeln umgeben, und das ist offenbar die dichteste mögliche Lagerung. Das dreieckige Flächengitter ist uns das Analogon der Raumgitter der Kristalle, und unsere Kugelschicht ist jetzt also, bildlich gesprochen, kristallinisch. Nach längerem Vibrieren wird die Zahl der Felder immer kleiner, bis ein Feld alle übrigen Felder aufgezehrt hat: die Schicht ist durch Umkristallisieren zu einem Kristall geworden. Dabei nimmt die Schicht mehr oder weniger rein die Gestalt eines regelmäBigen Sechsecks an: das ist ihre Kristallform. Wenn wir die Ordnung immer wieder zerstören, indem wir den Finger durchziehen, rekonstruiert sich nicht nur das Dreiecksgitter, sondern auch die Sechseckform immer wieder; die Schicht gravitiert also nach dem Dreieckgitter und nach der Sechseckform, weil das das Gitter gröBter Arbeit ist; das ist aber eben die Erscheinung, die O. Lehmann für unmöglich zu halten scheint. Wenn man allmählich immer stärker schüttelt, dann verwirrt sich ziemlich plötzlich gleichzeitig die ganze Ordnung und die Schicht wird ausgesprochen kreisrund: der Kristall ist geschmolzen und zu einer Flüssigkeit geworden. Die fortwährende Bewegung der Kugeln, die wir durch die fortwährende Vibration der Schüssel unterhalten, ist das Bild der kalorischen Bewegungen der Moleküle, die es zu einem vollkommenen Kontakt der Kugeln nicht kommen läBt. Wenn schon ein so grober Versuch so klare Analogien gibt, dann 
darf man wohl annehmen, daB auch die wirklichen Moleküle sehr leicht in die Gitterordnung gröBter Arbeit schnappen, nach der sie gravitieren.

Wir wollen nun zuerst das Raumgitter gröBter Arbeit betrachten, das sich ergibt, wenn wir annehmen, dab die Moleküle sich so verhalten, als wären sie Kugeln, deren Mittelpunkt der Sitz der Molekularanziehungen ist. Offenbar werden die Moleküle sich dann tetraedrisch ordnen, also so, wie wenn wir auf die erste dreieckig geordnete Schicht von Schrotkugeln in unserer Schüssel eine zweite, dritte... Schicht Kugeln legen, denn noch dichter können sich die Moleküle nicht lagern; das ist die Lagerung der gröBten Arbeit der Molekularkräfte. Die Mittelpunkte von je vier einander berührenden Kugeln bilden dann die Ecken eines Tetraeders. Wenn wir aber nicht die einander unmittelbar berührenden, sondern die zweitnächsten Kugeln ins Auge fassen, dann finden wir, da $B$ diese nicht tetraedrisch, sondern kubisch geordnet sind, d. h. je acht zweitnächste Kugeln geben in ihren Mittelpunkten die Ecken eines Würfels. Es gibt also in tetraedrischen Gefüge gröBter Arbeit drei aufeinander senkrechte Ebenen, denen parallel die je zweitnächsten Kugeln quadratisch geordnet sind. Aus diesem Gefüge lassen sich nun (wie das denn in der zitierten Arbeit auch geschieht) alle Formen des tesseralen Systems ableiten, wenn wir das Prinzip gröBter Arbeit auch auf die Oberfläche anwenden, und die Schnittlinien jener drei Ebenen sind die kristallographischen Achsen der Formen. Hiermit ist aber das tesserale Raumgitter als Raumgitter gröBter Arbeit nachgewiesen, das sich bei genügender Beweglichkeit der Moleküle nach jeder Strömung selbsttätig rekonstruieren muB. Eine Schrotmenge in einer Schale nimmt tatsächlich nach längerem Schütteln immer wieder dieses tetraedrische bez. kubische Gefüge an.

Wir finden leicht das Analoge für die übrigen Kristall. systeme; wir werden finden, daB sich in ihnen die Moleküle so verhalten, als wären sie nicht Kugeln, sondern Ellipsoide, und das soll gezeigt werden.

Wenn wir aus tetraedrisch gefügten Kugeln ein Oktaeder bauen, dann erscheinen die Kugeln in jeder Fläche dreieckig geordnet, d. h. sie liegen in Reihen, die den Dreieckseiten 
parallel liegen. ${ }^{1}$ ) Wenn wir nun das ganze Gefüge in vertikaler Richtung gestreckt denken, dann verwandeln sich die Kugeln in Rotationsellipsoide mit vertikalen Hauptachsen, aber das tetraedrische Gefüge bleibt vollkommen bewahrt; aus dem Oktaeder ist aber eine quadratische Pyramide geworden. Wenn wir das ganze Gebilde auch noch in einer horizontalen Achsenrichtung strecken, dann werden die Rotationsellipsoide zu dreiachsigen Ellipsoiden, deren Achsen den kristallographischen Achsen parallel liegen, und das ganze Gebilde wird zu einer rhombischen Pyramide. Das quadratische Raumgitter beruht also auf zweiachsigen, das rhombische Raumgitter auf dreiachsigen Ellipsoiden, beide aber sind Raumgitter größter Molekulararbeit, die sich selbsttätig rekonstruieren, denn sie haben tetraedrisches Gefüge.

Wenn wir nun mit der rhombischen Pyramide eine Deformation vornehmen, die in der Technik als gleitende Deformation bezeichnet wird, diese aber in der Richtung der einen Horizontalachse vornehmen, dann wird aus der rhombischen Pyramide eine monokline Pyramide. Die Ellipsoide bleiben bei dieser Deformation Ellipsoide, aber nur eine Ellipsoidachse fällt jetzt mit einer kristallographischen Achse zusammen, und zwar mit der Achse, die auf den beiden anderen senkrecht steht. In meiner zitierten Arbeit ist nun nachgewiesen, daB dieses Gefüge nur dann ein Gefüge gröBter Arbeit ist, wenn die Molekularkräfte sich so verhalten, als säBen sie nicht im Mittelpunkt der Moleküle, sondern in zwei getrennten Punkten der groben Achse, also etwa in zwei Brennpunkten oder in den Polen.

Wenn wir endlich die monokline Pyramide gleitend noch so deformieren, daB die kristallographischen Achsen lauter schiefe Winkel miteinander bilden, dann wird aus der monoklinen Pyramide eine trikline Pyramide, keine Ellipsoidachse fällt aber mehr mit einer kristallographischen Achse zusammen. Es läßt sich nachweisen, daB ein solches Gefüge dann ein Gefüge gröBter Arbeit ist, wenn die Molekularkräfte sich so verhalten, als säßen sie weder im Mittelpunkt, noch in der

1) Wenn wir die Ecken des Oktaeders schtchtenweise abtragen, erblicken wir die quadratisch geordneten Kugeln. 
großen Achse, sondern als gäbe es am Ellipsoid (Molekül) mehrere regellos verteilte Attraktionspole.

Wir sehen, daB jedes Kristallsystem auf anderartigen Molekülen beruht, und zwar liegen die Unterschiede teils an der Form der Moleküle, teils an der Zahl und Lage der Attraktionspole. Hier muB aber einem MiBverständnis vorgebeugt werden. In einem wirklichen Kristall sind die Moleküle nicht in dauerndem Kontakt, sondern sie sind auseinander gerückt und haben Spielraum für ihre kalorischen Schwingungen. Da kommen denn die Einzelheiten der Form nicht zur Geltung, und beispielsweise würde ein Molekülkomplex, der ähnlich einer Brombeere aus einem Zentralmolekül und zwölf umbüllenden Molekülen besteht, so wirken, als wäre es eine Kugel und würde tesserale Kristallformen geben. Da liegt es denn nahe, die Eillipsoide überhaupt als Molekülkomplexe anzusehen. Zwei gekuppelte Moleküle würden sich wie ein Rotationsellipsoid verhalten; drei gekuppelte Moleküle verhalten sich je nach Umständen wie ein Ring (es läßt sich zeigen, daB das hexagonale System am einfachsten aus Ringen von je drei gekuppelten Molekülen erklärt werden hann), oder wie ein Rotationsellipsoid mit kurzer Hauptachse. Vier tetraedrisch gekuppelte Moleküle verhalten sich wie eine Kugel; wenn sie aber in einer Ebene rhombisch geordnet sind, verhalten sie sich wie ein dreiachsiges Ellipsoid etc. Jetzt sind wir aber am Ziele: die kristallographische Polymorphie eines Stoffs läßt sich so erklären, dab der Stoff in verschiedenen Kristallsystemen verschiedene Molekülkomplexe bildet.

Die schönsten Argumente für diese Auffassung bietet 0. Lehmann selber. Vor etwa 15 Jahren demonstrierte o. Lehmann in Wien viele Stoffe, die mit steigender Temperatur das Kristallsystem wechselten. Es liegt die Vermutung nahe, da $B$ der Stoff bei tiefster Temperatur die molekülreichsten Komplexe bildete, und daB diese Komplexe mit steigender Temperatur immer weiter dissoziiert wurden, bis sie sich bei höchster Temperatur in Einzelmoleküle auflösten, die wir als Kugeln auffassen. Ich war nun überrascht, wie oft die Reihenfolge der Systeme offenbar dieser Auffassung entsprach, namentlich wie oft die Stoffe bei höchster Temperatur tesserale Formen zeigten, die eben Kugelmolekülen oder Einzelmolekülen entsprechen. 
398 K. Fuchs. Bemerkungen zu O. Lehmann: Kontinuität etc.

Das schönste Argument für die Aulfassung, daß die Kristalle im allgemeinen aus Ellipsoiden aufgebaut sind, liefert das optische Verhalten der Kristalle. Wenn man nämlich annimmt, daB die Achsen des optischen Elastizitütsellipsoides, aus den bekanntlich die Doppelbrechung erklärt wird, mit den geometrischen Achsen der Molekülellipsoide im Kristall der Richtung nach zusammenfallen, dann kann man aus dem von mir angenommenen Bau der Kristalle a priori bestimmen, welche Kristallsysteme Doppelbrechung zeigen müssen, welche einachsig, welche zweiachsig sein müssen, und welche Lage die optischen Achsen zu den kristallographischen Achsen haben müssen. Es ergibt sich nun, wie in meiner zitierten Arbeit gezeigt ist, eine vollkommene Übereinstimmung mit den beobachteten Tatsachen: die Kristalle verhalten sich optisch tatsächlich gerade so, wie es aus meiner Theorie des Kristallgefüges folgt.

(Eingegangen 31. August 1906.) 